IoAnNis K. Argyros (Lawton, OK)

\title{
ON THE SOLUTION AND APPLICATIONS OF GENERALIZED EQUATIONS USING NEWTON'S METHOD
}

Abstract. We provide local and semilocal convergence results for Newton's method when used to solve generalized equations. Using Lipschitz as well as center-Lipschitz conditions on the operators involved instead of just Lipschitz conditions we show that our Newton-Kantorovich hypotheses are weaker than earlier sufficient conditions for the convergence of Newton's method. In the semilocal case we provide finer error bounds and a better information on the location of the solution. In the local case we can provide a larger convergence radius. Our results apply to generalized equations involving single as well as multivalued operators, which include variational inequalities, nonlinear complementarity problems and nonsmooth convex minimization problems.

1. Introduction. In this study we are concerned with the problem of approximating a locally unique solution $x^{*}$ of the generalized equation

$$
F(x)+G(x) \ni 0,
$$

where $F: D_{0} \subseteq D \subseteq H \rightarrow H$ is a continuous operator which is Fréchetdifferentiable at each point of the interior $D_{0}$ of a closed convex subset $D$ of a Hilbert space $H$ with values in $H$, and $G$ is a multivalued maximal monotone operator from $H$ into $H$ (to be made precise later) [3], [9], [10].

The generalized Newton iteration

$$
F^{\prime}\left(x_{n}\right)\left(x_{n+1}\right)+G\left(x_{n+1}\right) \ni F^{\prime}\left(x_{n}\right)\left(x_{n}\right)-F\left(x_{n}\right) \quad(n \geq 0)
$$

has already been used to generate a sequence approximating $x^{*}$. In particular Uko [11], [12] has provided local and semilocal convergence results for method (2) as well as a procedure for the computation of the inner-iterative

2000 Mathematics Subject Classification: 65G99, 65J15, 47H17, 49M15.

Key words and phrases: Hilbert space, generalized equation, Newton's method, Lipschitz conditions, Newton-Kantorovich hypothesis, local-semilocal convergence theorems, coercivity, multivalued maximal monotone operator, radius of convergence. 
procedures for the computation of the generalized iterates $x_{n}(n \geq 0)$. This way he extended the classical Newton-Kantorovich results nonsmooth generalized equations. His results extend earlier work on nonsmooth equations [5], [7], [9], [10]. As in the classical cases Uko used Lipschitz differentiability conditions on $F^{\prime}$ and the maximality properties of $G$.

Here using a combination of center-Lipschitz and Lipschitz conditions we provide local and semilocal convergence results for method (2) with the following advantages over earlier works and in particular [12]:

(a) our results hold whenever the corresponding ones in [12] hold but not vice versa;

(b) in the semilocal case our Newton-Kantorovich hypotheses sufficient for the convergence of (2) are weaker than the corresponding one in $[12]$;

(c) our error bounds on the distances $\left\|x_{n+1}-x_{n}\right\|,\left\|x_{n}-x^{*}\right\|$ are finer and the information on the location of the solution $x^{*}$ more precise;

(d) in the local case and under weaker hypotheses our convergence radius can be larger. This observation is very important in computational mathematics (see also Remark 3).

Examples of special cases of (1) can be found in [1]-[3], [7]-[15] and the references there.

2. Semilocal analysis of method (2). Throughout this section we assume that

$$
\begin{aligned}
\left\|F^{\prime}(x)-F^{\prime}(y)\right\| & \leq q\|x-y\|, \\
\left\|F^{\prime}(x)-F^{\prime}\left(x_{0}\right)\right\| & \leq q_{0}\left\|x-x_{0}\right\|,
\end{aligned}
$$

for all $x, y \in D_{0}$ and some fixed $x_{0} \in D_{0}$. Moreover, $G$ is a nonempty subset of $H \times H$ so that there exists $a \geq 0$ such that

$$
[x, y] \in G \text { and }[v, w] \in G \Rightarrow\langle w-y, v-x\rangle \geq a\|x-v\|^{2},
$$

and which is not contained in any larger subset of $H \times H$.

We will use Lemma 2.2 from [12, p. 256]:

Lemma 1. Let $G$ be a maximal monotone operator satisfying (5), and let $M$ be a bounded linear operator from $H$ into $H$. If there exists $c \in \mathbb{R}$ such that $c>-a$, and

$$
\langle M(x), x\rangle \geq c\|x\|^{2} \quad \text { for all } x \in H,
$$

then for any $b \in H$ there exists a unique $z \in H$ such that

$$
M(z)+G(z) \ni b .
$$

We provide the following result on majorizing sequences: 
Lemma 2. Assume that there exist parameters $L, L_{0}, \eta \geq 0$ with $L_{0} \leq L$, and $\delta \in[0,1]$ such that

$$
h_{\delta}=\left(\delta L_{0}+L\right) \eta \leq \delta .
$$

Then the sequence $\left\{t_{n}\right\}(n \geq 0)$ given by

$$
t_{0}=0, \quad t_{1}=\eta, \quad t_{n+2}=t_{n+1}+\frac{L\left(t_{n+1}-t_{n}\right)^{2}}{2\left(1-L_{0} t_{n+1}\right)} \quad(n \geq 0)
$$

is nondecreasing, bounded above by $t^{* *}=2 \eta /(2-\delta)$ and converges to some $t^{*}$ such that

$$
0 \leq t^{*} \leq t^{* *}
$$

Moreover, the following error bounds hold for all $n \geq 0$ :

$$
0 \leq t_{n+2}-t_{n+1} \leq \frac{\delta}{2}\left(t_{n+1}-t_{n}\right) \leq\left(\frac{\delta}{2}\right)^{n+1} \eta .
$$

Proof. The result clearly holds if either $\delta, L$ or $\eta$ is zero. Assume $\delta, L, \eta$ $\neq 0$. We must show that for all $n \geq 0$,

$$
L\left(t_{n+1}-t_{n}\right)+\delta L_{0} t_{n+1} \leq \delta, \quad t_{n+1}-t_{n} \geq 0, \quad 1-L_{0} t_{n+1}>0 .
$$

Estimate (11) then follows immediately from (9) and (12). We use induction on $n$. For $n=0$ we have

$$
L\left(t_{1}-t_{0}\right)+\delta L_{0} t_{1}=L \eta+\delta L_{0} \eta \leq \delta, \quad t_{1} \geq t_{0}, \quad 1-L_{0} \eta>0
$$

by (8). But then (9) gives

$$
0 \leq t_{2}-t_{1} \leq \frac{\delta}{2}\left(t_{1}-t_{0}\right) .
$$

Assume (11) and (12) hold for all $n \leq k+1$. Then

$$
\begin{aligned}
& L\left(t_{k+2}-t_{k+1}\right)+\delta L_{0} t_{k+2} \\
\leq & L \eta\left(\frac{\delta}{2}\right)^{k+1} \\
& \quad+\delta L_{0}\left[t_{1}+\frac{\delta}{2}\left(t_{1}-t_{0}\right)+\left(\frac{\delta}{2}\right)^{2}\left(t_{1}-t_{0}\right)+\ldots+\left(\frac{\delta}{2}\right)^{k+1}\left(t_{1}-t_{0}\right)\right] \\
\leq & L \eta\left(\frac{\delta}{2}\right)^{k+1}+\delta L_{0} \eta \frac{1-(\delta / 2)^{k+2}}{1-\delta / 2} \\
= & L \eta\left(\frac{\delta}{2}\right)^{k+1}+\frac{2 \delta L_{0} \eta}{2-\delta}\left[1-\left(\frac{\delta}{2}\right)^{k+2}\right] \\
= & \left\{L\left(\frac{\delta}{2}\right)^{k+1}+\frac{2 L_{0} \delta}{2-\delta}\left[1-\left(\frac{\delta}{2}\right)^{k+2}\right]\right\} \eta .
\end{aligned}
$$


By (8) and (13) it suffices to show

$$
L\left(\frac{\delta}{2}\right)^{k+1}+\frac{2 L_{0} \delta}{2-\delta}\left[1-\left(\frac{\delta}{2}\right)^{k+2}\right] \leq L+\delta L_{0},
$$

or

$$
\delta L_{0}\left\{\frac{2}{2-\delta}\left(1-\left(\frac{\delta}{2}\right)^{k+2}\right)-1\right\} \leq L\left[1-\left(\frac{\delta}{2}\right)^{k+1}\right]
$$

or

$$
\left[\frac{L_{0} \delta^{2}}{2-\delta}-L\right]\left[1-\left(\frac{\delta}{2}\right)^{k+1}\right] \leq 0,
$$

or

$$
\frac{L_{0} \delta^{2}}{2-\delta} \leq L,
$$

which is true by the choice of $\delta$. Hence, the first estimate in (12) holds for all $n \geq 0$. We must also show that

$$
t_{k} \leq t^{* *}
$$

For $k=0,1,2$ we have

$$
t_{0}=0 \leq t^{* *}, \quad t_{1}=\eta \leq t^{* *}, \quad t_{2} \leq \eta+\frac{\delta}{2} \eta=\frac{2+\delta}{2} \eta \leq t^{* *} .
$$

It follows from (11) that for all $k \geq 0$,

$$
\begin{aligned}
t_{k+2} & \leq t_{k+1}+\frac{\delta}{2}\left(t_{k+1}-t_{k}\right) \leq t_{k}+\frac{\delta}{2}\left(t_{k}-t_{k-1}\right)+\frac{\delta}{2}\left(t_{k+1}-t_{k}\right) \\
& \leq \ldots \leq t_{1}+\frac{\delta}{2}\left(t_{1}-t_{0}\right)+\ldots+\left(\frac{\delta}{2}\right)\left(t_{k}-t_{k-1}\right)+\frac{\delta}{2}\left(t_{k+1}-t_{k}\right) \\
& \leq \eta+\frac{\delta}{2} \eta+\left(\frac{\delta}{2}\right)^{2} \eta+\ldots+\left(\frac{\delta}{2}\right)^{k+1} \eta \\
& =\left[1+\frac{\delta}{2}+\left(\frac{\delta}{2}\right)^{2}+\ldots+\left(\frac{\delta}{2}\right)^{k+1}\right] \eta \\
& =\frac{1-(\delta / 2)^{k+2}}{1-\delta / 2} \eta<\frac{2}{2-\delta} \eta=t^{* *} .
\end{aligned}
$$

Moreover, we have

$$
L_{0} t_{k+2}<\frac{2 L_{0} \eta}{2-\delta} \leq 1
$$

(by (8)). Hence, the sequence $\left\{t_{n}\right\}$ is bounded above by $t^{* *}$. It also follows from (9) that $\left\{t_{n}\right\}$ is nondecreasing and so it converges to some $t^{*}$ satisfying (10).

That completes the proof of Lemma 2. 
REMARK 1. It follows immediately from the proof of Lemma 2 that condition (8) can be replaced by the weaker

$$
h_{\delta} \leq \delta, \quad \frac{L_{0} \delta^{2}}{2-\delta} \leq L, \quad \frac{2 L_{0} \eta}{2-\delta} \leq 1, \quad \delta \in[0,2) .
$$

We present the main semilocal convergence theorem for method (2) using Lipschitz conditions (3) and center-Lipschitz conditions (4).

Theorem 1. Let $F$ and $G$ satisfy (3), (4) and (5), (6) respectively, for $M=F^{\prime}\left(x_{0}\right)$. For $x_{0} \in D_{0}$ assume there exists $y_{0} \in H$ such that $G\left(x_{0}\right) \ni y_{0}$ and $\left\|F\left(x_{0}\right)+y_{0}\right\| \leq b_{0}$ for $b_{0}>0$. Moreover suppose (8) holds for

$$
L_{0}=\frac{q_{0}}{c_{0}+a}, \quad c_{0}=c, \quad L=\frac{q}{c_{0}+a}, \quad \eta=\frac{b_{0}}{c_{0}+a},
$$

and

$$
\bar{U}\left(x_{0}, t^{*}\right)=\left\{x \in X:\left\|x-x_{0}\right\| \leq t^{*}\right\} \subseteq D .
$$

Then the sequence $\left\{x_{n}\right\}$ ( $\left.n \geq 0\right)$ generated by generalized Newton's method (2) is well defined, remains in $\bar{U}\left(x_{0}, t^{*}\right)$ for all $n \geq 0$, and converges to a unique solution $x^{*}$ of equation (1) in $\bar{U}\left(x_{0}, t^{*}\right)$. Moreover the following error bounds hold for all $n \geq 0$ :

$$
\begin{aligned}
\left\|x_{n+1}-x_{n}\right\| & \leq t_{n+1}-t_{n}, \\
\left\|x_{n}-x^{*}\right\| & \leq t^{*}-t_{n},
\end{aligned}
$$

where $\left\{t_{n}\right\}$ is given by $(9)$.

Proof. We use induction on $k=0,1,2, \ldots$ to show:

$$
\begin{aligned}
& x_{k} \in \bar{U}\left(x_{0}, t^{*}\right), \\
& \left\|x_{k+1}-x_{k}\right\| \leq t_{k+1}-t_{k}, \\
& \bar{U}\left(x_{k+1}, t^{*}-t_{k+1}\right) \subseteq \bar{U}\left(x_{k}, t^{*}-t_{k}\right), \\
& \exists y_{k} \in H \text { such that } y_{k} \in G\left(x_{k}\right), \\
& \exists b_{k}>0 \text { such that }\left\|F\left(x_{k}\right)+y_{k}\right\| \leq b_{k}, \\
& \exists c_{k}>-a \text { such that }\left\langle F^{\prime}\left(x_{k}\right)(x), x\right\rangle \geq c_{k}\|x\|^{2} \text { for all } x \in H .
\end{aligned}
$$

The assertions (20), (23)-(25) are true if $k=0$ by the hypotheses of the theorem. It then follows from (25) and Lemma 1 that there exists a unique $x_{1} \in H$ satisfying (2). By (5), (6), (9), (16) and (2) we obtain in turn

$$
a\left\|x_{1}-x_{0}\right\|^{2}+\left\langle y_{0}+F\left(x_{0}\right)-F^{\prime}\left(x_{0}\right)\left(x_{0}-x_{1}\right), x_{1}-x_{0}\right\rangle \leq 0,
$$

hence

$$
a\left\|x_{1}-x_{0}\right\|^{2}+\left\langle F^{\prime}\left(x_{0}\right)\left(x_{1}-x_{0}\right), x_{1}-x_{0}\right\rangle \leq\left\langle-F\left(x_{0}\right)-y_{0}, x_{1}-x_{0}\right\rangle,
$$

and so

$$
\left\|x_{1}-x_{0}\right\| \leq a_{0}=\frac{b_{0}}{c_{0}+a}=t_{1}-t_{0}
$$


For every $z \in \bar{U}\left(x_{1}, t^{*}-t_{1}\right)$,

$$
\left\|z-x_{0}\right\| \leq\left\|z-x_{1}\right\|+\left\|x_{1}-x_{0}\right\| \leq t^{*}-t_{1}+t_{1}=t^{*}-t_{0},
$$

which implies $z \in \bar{U}\left(x_{0}, t^{*}-t_{0}\right)$. It follows from (27) and (28) that (21) and (22) hold for $k=0$. Given they hold for $n=0, \ldots, k$ and again using (25) and Lemma 1 we conclude that there exists a unique $x_{k+1} \in H$ satisfying (2),

$$
\begin{aligned}
\left\|x_{k+1}-x_{0}\right\| & \leq \sum_{i=1}^{k+1}\left\|x_{i}-x_{i-1}\right\| \\
& \leq \sum_{i=1}^{k+1}\left(t_{i}-t_{i-1}\right)=t_{k+1}-t_{0}=t_{k+1} \leq t^{*},
\end{aligned}
$$

$$
\left\|x_{k}+\theta\left(x_{k+1}-x_{k}\right)-x_{0}\right\| \leq t_{k}+\theta\left(t_{k+1}-t_{k}\right)<t^{*}, \quad \theta \in[0,1] .
$$

Hence (20) holds if $k$ is replaced by $k+1$. As in (26) we obtain in turn

$$
a\left\|x_{k+1}-x_{k}\right\|^{2}+\left\langle y_{k}+F\left(x_{k}\right)-F^{\prime}\left(x_{k}\right)\left(x_{k}-x_{k+1}\right), x_{k+1}-x_{k}\right\rangle \leq 0,
$$

so

$$
\begin{aligned}
a\left\|x_{k+1}-x_{k}\right\|^{2}+\left\langle F^{\prime}\left(x_{k}\right)\left(x_{k+1}-x_{k}\right)\right. & \left., x_{k+1}-x_{k}\right\rangle \\
& \leq\left\langle-F\left(x_{k}\right)-y_{k}, x_{k+1}-x_{k}\right\rangle,
\end{aligned}
$$

and thus

$$
\left\|x_{k+1}-x_{k}\right\| \leq t_{k+1}-t_{k} .
$$

That is, (21) and (22) hold with $k$ replaced by $k+1$.

By (4) and (29) we get

$$
\left\|F^{\prime}\left(x_{k+1}\right)-F^{\prime}\left(x_{0}\right)\right\| \leq q_{0}\left\|x_{k+1}-x_{0}\right\| \leq q_{0} t_{k+1} .
$$

Set

$$
c_{k+1}=c_{0}-q_{0} t_{k}
$$

Then by hypothesis (8) we get

$$
c_{k+1}>-a .
$$

Therefore

$$
\left\langle F^{\prime}\left(x_{0}\right)(x)-F^{\prime}\left(x_{k+1}\right)(x), x\right\rangle \leq\left\|F^{\prime}\left(x_{0}\right)-F^{\prime}\left(x_{k+1}\right)\right\|\|x\|^{2} \leq q_{0} t_{k}\|x\|^{2}
$$

for all $x \in H$. Hence (25) holds with $k$ replaced by $k+1$.

Define

$$
y_{k+1}=-F\left(x_{k}\right)-F^{\prime}\left(x_{k}\right)\left(x_{k+1}-x_{k}\right) .
$$

Then (23) holds by (7) and 


$$
\begin{aligned}
\| F\left(x_{k+1}\right)+ & y_{k+1}\|\leq\| F\left(x_{k+1}\right)-F\left(x_{k}\right)-F^{\prime}\left(x_{k}\right)\left(x_{k+1}-x_{k}\right) \| \\
& =\left\|\int_{0}^{1}\left[F^{\prime}\left(x_{k}+\theta\left(x_{k+1}-x_{k}\right)\right)-F^{\prime}\left(x_{k}\right)\right]\left(x_{k+1}-x_{k}\right) d t\right\| \\
& \leq \frac{q}{2}\left\|x_{k+1}-x_{k}\right\|^{2}=b_{k+1},
\end{aligned}
$$

where

$$
a_{k}=\frac{b_{k}}{c_{k}+a} \quad(k \geq 0) .
$$

Thus for every $z \in \bar{U}\left(x_{k+1}, t^{*}-t_{k+1}\right)$, we have

$$
\begin{aligned}
\left\|z-x_{k}\right\| & \leq\left\|z-x_{k+1}\right\|+\left\|x_{k+1}-x_{k}\right\| \\
& \leq t^{*}-t_{k+1}+t_{k+1}-t_{k}=t^{*}-t_{k} .
\end{aligned}
$$

That is,

$$
z \in \bar{U}\left(x_{k}, t^{*}-t_{k}\right)
$$

The induction for (20)-(25) is now complete.

Lemma 2 implies that $\left\{t_{n}\right\}$ is a Cauchy sequence. By (9) and (32) it follows that $\left\{x_{n}\right\}$ is also a Cauchy sequence, and so it converges to some $x^{*} \in \bar{U}\left(x_{0}, t^{*}\right)$ (since $\bar{U}\left(x_{0}, t^{*}\right)$ is a closed set). By letting $m \rightarrow \infty$ in

$$
\left\|x_{k+m}-x_{k}\right\| \leq \sum_{i=k}^{k+m-1}\left\|x_{i+1}-x_{i}\right\| \leq \sum_{i=k}^{k+m-1}\left(t_{i+1}-t_{i}\right)=t_{k+m}-t_{k}
$$

we obtain (19). Moreover, since $\lim _{k \rightarrow \infty} x_{k+1}=x^{*}$ and

$$
\begin{gathered}
\lim _{k \rightarrow \infty}\left[F^{\prime}\left(x_{k}\right)\left(x_{k}-x_{k+1}\right)-F\left(x_{k}\right)\right]=-F\left(x^{*}\right), \\
G\left(x_{k+1}\right) \ni F^{\prime}\left(x_{k}\right)\left(x_{k+1}-x_{k}\right)-F\left(x_{k}\right),
\end{gathered}
$$

it follows that $G\left(x^{*}\right) \ni-F\left(x^{*}\right)$. Hence $x^{*}$ is a solution of (1).

Finally, to show uniqueness in $\bar{U}\left(x_{0}, t^{*}\right)$, assume there exists a solution $y^{*} \in \bar{U}\left(x_{0}, t^{*}\right)$. Then we obtain

$$
\begin{aligned}
a\left\|x_{k+1}-y^{*}\right\|^{2}+\left\langle F^{\prime}\left(x_{k}\right)\right. & \left.\left(x_{k+1}-y^{*}\right), x_{k+1}-y^{*}\right\rangle \\
& \leq\left\langle F\left(y^{*}\right)-F\left(x_{k}\right)-F^{\prime}\left(x_{k}\right)\left(y^{*}-x_{k}\right), x_{k+1}-y^{*}\right\rangle
\end{aligned}
$$

or equivalently (as in (31))

$$
\left\|x_{k+1}-y^{*}\right\| \leq \frac{q}{2\left(c_{k}+a\right)}\left\|x_{k}-y^{*}\right\|^{2}<\left\|x_{k}-y^{*}\right\|
$$

(since $\frac{q}{2\left(c_{k}+a\right)}\left\|x_{k}-y^{*}\right\|<1$ by (8)). Hence we get $x^{*}=\lim _{k \rightarrow \infty} x_{k}=y^{*}$.

That completes the proof of Theorem 1.

REMARK 2 . Note that $t^{*}$ can be replaced by $2 \eta /(2-\delta)$ in condition (17). 
REMARK 3. To compare our Theorem 1 with earlier ones, in particular with Theorem 2.11 of [12], we define the scalar function $p$ by

$$
p(s)=\frac{L}{2} s^{2}-s+a_{0},
$$

where $L$ is given by (16). Uko's Newton-Kantorovich hypothesis (see (2.14) in [12]) becomes

$$
h=2 L a_{0} \leq 1,
$$

whereas ours for $\delta=1$ reduces to

$$
h_{1}=\left(L+L_{0}\right) a_{0} \leq 1 .
$$

But in general

$$
L_{0} \leq L
$$

Hence (45) always implies (46) but not vice versa unless $L=L_{0}$. If strict inequality holds in (47) then (46) may hold but not (45). In Example 3 that follows we show that $L / L_{0}$ may be arbitrarily large. Moreover define a sequence $\left\{s_{n}\right\}$ by

$$
s_{n+1}=s_{n}+\frac{\frac{L}{2} s_{n}^{2}-s_{n}+a_{0}}{1-L s_{n}}, \quad s_{0}=0(n \geq 0),
$$

and set

$$
s^{*}=\lim _{n \rightarrow \infty} s_{n} .
$$

Then it is known [3], [6] that

$$
\begin{aligned}
s^{*} & =\frac{1-\sqrt{1-2 L_{0} a_{0}}}{L}, \\
s_{n+1}-s_{n} & =-\frac{p\left(s_{n}\right)}{p^{\prime}\left(s_{n}\right)}=\frac{\frac{L}{2}\left(s_{n}-s_{n-1}\right)^{2}}{1-L s_{n}} \quad(n \geq 1), \\
s^{*}-s_{n+1} & =\frac{\frac{L}{2}\left(s^{*}-s_{n}\right)^{2}}{1-L s_{n}} \leq \frac{1}{L 2^{n+1}} h^{2^{n+1}} \quad(n \geq 0) .
\end{aligned}
$$

Uko essentially showed error bounds (18) and (19) with the sequence $\left\{s_{n}\right\}$ and point $s^{*}$ replacing $\left\{t_{n}\right\}$ and $t^{*}$ respectively.

That is, for all $n \geq 0$,

$$
\begin{aligned}
\left\|x_{n+1}-x_{n}\right\| & \leq s_{n+1}-s_{n} \\
\left\|x_{n}-x^{*}\right\| & \leq s^{*}-s_{n} .
\end{aligned}
$$

We show that our error bounds are finer and the location of the solution $x^{*}$ more precise:

Proposition 1. Under the hypotheses of Theorem $1\left(\right.$ for $\left.\ell_{0}<\ell\right)$ and (45) the following error bounds hold:

$$
t_{n+1}<s_{n+1} \quad(n \geq 1),
$$




$$
\begin{array}{cl}
t_{n+1}-t_{n}<s_{n+1}-s_{n} & (n \geq 1) \\
t^{*}-t_{n} \leq s^{*}-s_{n} & (n \geq 0) \\
t^{*} \leq s^{*}, & \\
0 \leq t_{n+1}-t_{n} \leq \alpha^{2^{n-1}}\left(s_{n+1}-s_{n}\right) \quad(n \geq 1), \quad \alpha=\frac{1-\ell \eta}{1-\ell_{0} \eta} \in[0,1)
\end{array}
$$$$
0 \leq t^{*}-t_{n} \leq \alpha^{2^{n-1}}\left(s^{*}-s_{n}\right) \quad(n \geq 1) .
$$

Moreover, $t_{n}=s_{n}(n \geq 0)$ if $\ell=\ell_{0}$.

Proof. We use induction on $n$ to show (53) and (54) first. For $n=0$ in (9) we obtain

$$
t_{2}-\eta=\frac{\ell \eta^{2}}{2\left(1-\ell_{0} \eta\right)} \leq \frac{\ell \eta^{2}}{2(1-\ell \eta)}=s_{2}-s_{1}
$$

and so

$$
t_{2}<s_{2}
$$

Assume that

$$
t_{k+1}<s_{k+1}, \quad t_{k+1}-t_{k}<s_{k+1}-s_{k} \quad(k \leq n+1) .
$$

Using (9) and (48) we get

$$
t_{k+2}-t_{k+1}=\frac{\frac{\ell}{2}\left(t_{k+1}-t_{k}\right)^{2}}{1-\ell_{0} t_{k+1}}<\frac{\frac{\ell}{2}\left(s_{k+1}-s_{k}\right)^{2}}{1-\ell s_{k+1}}=s_{k+2}-s_{k+1} .
$$

For $m \geq 0$, we obtain

$$
\begin{aligned}
t_{k+m} & -t_{k} \\
& <\left(t_{k+m}-t_{k+m-1}\right)+\left(t_{k+m-1}-t_{k+m-2}\right)+\ldots+\left(t_{k+1}-t_{k}\right) \\
& <\left(s_{k+m}-s_{k+m-1}\right)+\left(s_{k+m-1}-s_{k+m-2}\right)+\ldots+\left(s_{k+1}-s_{k}\right) \\
& <s_{k+m}-s_{k} .
\end{aligned}
$$

By letting $m \rightarrow \infty$ in (59) we obtain (55). For $n=1$ in (55) we get (56).

Finally, (57) and (58) follow easily from (9) and (48). Note also that (57) holds as a strict inequality if $n \geq 2$.

That completes the proof of Proposition 1.

REMARK 4. We now complete this section with three numerical examples when $G=0$ on $D$. In the first one, hypothesis (45) fails whereas (46) holds. In the second example, we show that estimates (18), (19) compare favorably with $(18)^{\prime},(19)^{\prime}$, respectively. In the third one, we show that $L / L_{0}$ can be arbitrarily large.

Example 1 . Let $H=\mathbb{R}, D=[\sqrt{2}-1, \sqrt{2}+1], x_{0}=\sqrt{2}$ and define a function $F$ on $D$ by

$$
F(x)=\frac{1}{6} x^{3}-\left(\frac{2^{3 / 2}}{6}+.23\right) .
$$


Using (3)-(6) we obtain

$$
\begin{aligned}
a=0, \quad c=2, \quad a_{0} & =.23, \quad L=2.4142136, \quad L_{0}=1.914213562, \\
h & =2 L a_{0}=1.1105383>1,
\end{aligned}
$$

and by (8) for $\delta=1$,

$$
\left(L+L_{0}\right) a_{0}=.995538247<1 .
$$

That is, there is no guarantee that Newton's method $\left\{x_{n}\right\}$ starting at $x_{0}$ converges to a solution $x^{*}$ of equation $F(x)=0$, since (45) is violated. However since (62) holds, Theorem 1 guarantees the convergence of Newton's method to $x^{*}=1.614507018$.

Example 2. Let $H=\mathbb{R}, x_{0}=1.3, D=\left[x_{0}-2 \eta, x_{0}+2 \eta\right]$ and define a function $F$ on $D$ by

$$
F(x)=\frac{1}{3}\left(x^{3}-1\right) .
$$

As in Example 1 we obtain

$$
\begin{aligned}
a_{0} & =.236094674, \quad L=2.097265501, \quad L_{0}=1.817863519, \\
h & =2 L \eta=.990306428<1, \\
h_{1} & =\left(L+L_{0}\right) \eta=.92434111<1 \quad(\text { for } \delta=1), \\
t^{*} & =.369677842, \quad s^{*}=.429866445 .
\end{aligned}
$$

That is, we provide a better information on the location of the solution $x^{*}$ since

$$
\bar{U}\left(x_{0}, t^{*}\right) \subset \bar{U}\left(x_{0}, s^{*}\right) .
$$

Moreover using (2) and (63) we can tabulate the following results:

\section{Comparison table}

\begin{tabular}{ccccc}
\hline$x_{n}$ & Estimates (18) & Estimates (19) & Estimates (18) & Estimates (19)' \\
\hline$x_{1}=1.0639053254$ & .236094674 & .133583172 & .236094674 & .193771771 \\
$x_{2}=1.0037617275$ & .102400629 & .031182539 & .115780708 & .0779910691 \\
$x_{3}=1.0000140800$ & .028585756 & .002596783 & .053649732 & .024342893 \\
$x_{4}=1.0000000002$ & .002575575 & .000021208 & .020186667 & .004156226 \\
$n=5$ & .000021207 & .000000001 & .003987206 & .00016902 \\
$n=6$ & .000000001 & 0 & .000166761 & .000002259 \\
\hline
\end{tabular}

ExAmple 3 . Let $H=\mathbb{R}, x_{0}=0$ and define a function $F$ on $\mathbb{R}(G=0)$ by

$$
F(x)=b_{0} x+b_{1}+b_{2} \sin e^{b_{3} x},
$$

where $b_{i}, i=0,1,2,3$, are given parameters. It can easily be seen that for $b_{3}$ large and $b_{2}$ sufficiently small, $L / L_{0}$ may be arbitrarily large. That is, (46) may be satisfied but not (45). 
3. Local analysis for method (2). Throughout this section we assume that

$$
\left\|F^{\prime}(x)-F^{\prime}\left(x^{*}\right)\right\| \leq \ell\left\|x-x^{*}\right\| \quad \text { for all } x \in D_{0} .
$$

We can show the following local result for method (2):

Theorem 2. Let $G$ be a maximal monotone operator satisfying (5). Suppose (6) holds for $M=F^{\prime}\left(x^{*}\right)$ and the generalized equation (1) has a solution $x^{*}$ in $D_{0}$ such that

$$
\bar{U}\left(x^{*}, r^{*}\right) \subseteq D_{0},
$$

where

$$
r^{*}=\frac{2}{3 \ell}(a+c) .
$$

Then the sequence $\left\{x_{n}\right\}(n \geq 0)$ generated by generalized Newton's method (2) is well defined, remains in $U\left(x^{*}, r^{*}\right)$ for all $n \geq 0$, and converges to $x^{*}$ provided that $x_{0} \in U\left(x^{*}, r^{*}\right)$.

Moreover the following error bounds hold for all $n \geq 0$ :

$$
\begin{aligned}
\left\|x_{1}-x^{*}\right\| & \leq \frac{\ell}{2(a+c)}\left\|x_{0}-x^{*}\right\|^{2}, \\
\left\|x_{n+1}-x^{*}\right\| & \leq \frac{\ell}{2\left(a+c-\ell\left\|x_{0}-x^{*}\right\|\right)}\left\|x_{n}-x^{*}\right\|^{2} \leq d_{0} d^{2^{n}} \quad(n \geq 1),
\end{aligned}
$$

where

$$
\begin{aligned}
d_{0} & =\frac{2\left(a+c-\ell\left\|x_{0}-x^{*}\right\|\right)}{\ell}, \\
d & =\left\|x_{0}-x^{*}\right\| d_{0}^{-1} .
\end{aligned}
$$

Proof. We first establish the existence of solution $x_{1}$. Using (6) and (65) we obtain in turn for all $x \in H$

$$
\begin{aligned}
\left\langle\left[F^{\prime}\left(x^{*}\right)-F^{\prime}\left(x_{0}\right)\right](x), x\right\rangle & \leq\left\|F^{\prime}\left(x^{*}\right)-F^{\prime}\left(x_{0}\right)\right\|\|x\|^{2} \\
& \leq \ell\left\|x_{0}-x^{*}\right\|\|x\|^{2}
\end{aligned}
$$

so

$$
c\|x\|^{2}-\ell\left\|x_{0}-x^{*}\right\|\|x\|^{2} \leq\left\langle F^{\prime}\left(x_{0}\right)(x), x\right\rangle,
$$

that is,

$$
\left(c-\ell\left\|x_{0}-x^{*}\right\|\right)\|x\|^{2} \leq\left\langle F^{\prime}\left(x_{0}\right)(x), x\right\rangle .
$$

It follows by the choice of $x_{0}$ that

$$
-\ell\left\|x_{0}-x^{*}\right\|>-a \text {. }
$$

Hence by Lemma 1, $x_{1}$ exists, and solves (1). By (5) we obtain

$$
a\left\|x_{1}-x^{*}\right\|^{2} \leq\left\langle F\left(x^{*}\right)-F\left(x_{0}\right)-F^{\prime}\left(x_{0}\right)\left(x_{1}-x_{0}\right), x_{1}-x^{*}\right\rangle
$$


so that

$$
\begin{aligned}
& a\left\|x_{1}-x^{*}\right\|^{2}+\left\langle F^{\prime}\left(x_{0}\right)\left(x_{1}-x^{*}\right), x_{1}-x^{*}\right\rangle \\
& \leq\left\langle F\left(x^{*}\right)-F\left(x_{0}\right)-F^{\prime}\left(x_{0}\right)\left(x^{*}-x_{0}\right), x_{1}-x^{*}\right\rangle
\end{aligned}
$$

or equivalently

$$
(a+c)\left\|x_{1}-x^{*}\right\| \leq \frac{\ell}{2}\left\|x_{0}-x^{*}\right\|^{2},
$$

which shows (68), $x_{1} \in U\left(x^{*}, r^{*}\right)$, and in particular

$$
\left\|x_{1}-x^{*}\right\|<\left\|x_{0}-x^{*}\right\| \text {. }
$$

Assume $x_{k} \in U\left(x^{*}, r^{*}\right), x_{k}$ solves (1) and

$$
\left\|x_{k}-x^{*}\right\|<\left\|x_{0}-x^{*}\right\| \quad(k \geq 1) .
$$

As in (74) we get in turn

$$
\left\langle\left[F^{\prime}\left(x^{*}\right)-F^{\prime}\left(x_{k}\right)\right](x), x\right\rangle \leq\left\|F^{\prime}\left(x^{*}\right)-F^{\prime}\left(x_{k}\right)\right\|\|x\|^{2} \leq \ell\left\|x^{*}-x_{k}\right\|\|x\|^{2},
$$

so

$$
\left\langle F^{\prime}\left(x^{*}\right)(x), x\right\rangle-\left\langle F^{\prime}\left(x_{k}\right)(x), x\right\rangle \leq \ell\left\|x_{0}-x^{*}\right\|\|x\|^{2}
$$

and hence

$$
\left(c-\ell\left\|x_{0}-x^{*}\right\|\right)\|x\|^{2} \leq\left\langle F^{\prime}\left(x_{k}\right)(x), x\right\rangle,
$$

which establishes the existence of $x_{k+1}$. Moreover by (5) we get

$$
a\left\|x_{k+1}-x^{*}\right\|^{2} \leq\left\langle F\left(x^{*}\right)-F\left(x_{k}\right)-F^{\prime}\left(x_{k}\right)\left(x_{k+1}-x_{k}\right), x_{k+1}-x^{*}\right\rangle,
$$

that is,

$$
\begin{aligned}
a\left\|x_{k+1}-x^{*}\right\|^{2}+\left\langle F^{\prime}\right. & \left.\left(x_{k}\right)\left(x_{k+1}-x^{*}\right), x_{k+1}-x^{*}\right\rangle \\
\leq & \left\langle F\left(x^{*}\right)-F\left(x_{k}\right)-F^{\prime}\left(x_{k}\right)\left(x^{*}-x_{k}\right), x_{k+1}-x^{*}\right\rangle
\end{aligned}
$$

and so

$$
\left(a+c-\ell\left\|x_{0}-x^{*}\right\|\right)\left\|x_{k+1}-x^{*}\right\| \leq \frac{\ell}{2}\left\|x_{k}-x^{*}\right\|^{2},
$$

which shows (69), $x_{k+1} \in U\left(x^{*}, r^{*}\right)$, and $\lim _{k \rightarrow \infty} x_{k}=x^{*}$.

That completes the proof of Theorem 2 .

REMARK 5. A local result similar to Theorem 2 is given in [12, Thm. 2.5] where the following conditions, stronger and more difficult to verify, are used:

$$
\left\|F^{\prime}(x)-F^{\prime}(y)\right\| \leq \gamma\|x-y\| \quad \text { for all } x \in D_{0},
$$

there exists $c_{1}>-a$ such that $\left\langle F^{\prime}(z)(x), x\right\rangle \geq c_{1}\|x\|^{2}$

$$
\text { for all } x \in H, z \in D_{0} \text {. }
$$

The coercivity condition (79) which implies $F^{\prime}(x)^{-1}$ exists for all $x \in D_{0}$ is rather strong, and may not hold in many problems occurring in applications. Note also that it is possible to obtain a larger convergence radius despite the fact that we use weaker conditions (see, e.g., Example 4 that follows). 
REMARK 6. As noted in [1]-[3], [4], [11]-[15] the local results obtained here can be used for projection methods such as Arnoldi's, the generalized minimum residual method (GMRES), the generalized conjugate residual method (GCR), for combined Newton/finite-difference projection methods, in connection with the mesh independence principle in order to develop the most efficient mesh refinement strategies, variational inequalities, nonlinear complementarity problems and nonsmooth convex minimization problems.

REMARK 7. The local results obtained here can also be used to solve equations of the form (1), where $F^{\prime}$ satisfies the autonomous differential equation [3], [6]:

$$
F^{\prime}(x)=T(F(x)),
$$

where $T: H \rightarrow H$ is a known continuous operator. Since $F^{\prime}\left(x^{*}\right)=T\left(F\left(x^{*}\right)\right)$ $=T(0)$, we can apply the results obtained here without actually knowing the solution $x^{*}$ of equation (1).

Example 4 . Let $H=\mathbb{R}, D=\bar{U}(0,1), G=0$ on $D$, and define $F$ by

$$
F(x)=e^{x}-1 .
$$

Then it can easily be seen that we can set $T(x)=x+1$ in (80). We obtain

$$
\begin{aligned}
F^{\prime}(x)-F^{\prime}\left(x^{*}\right) & =e^{x}-1=x+\frac{x^{2}}{2 !}+\ldots+\frac{x^{n}}{n !}+\ldots \\
& =\left(1+\frac{x}{2 !}+\ldots+\frac{x^{n-1}}{n !}+\ldots\right)\left(x-x^{*}\right),
\end{aligned}
$$

and for $x \in \bar{U}(0,1)$,

$$
\left\|F^{\prime}(x)-F^{\prime}\left(x^{*}\right)\right\| \leq(e-1)\left\|x-x^{*}\right\| .
$$

That is, $\ell=e-1$. Moreover $a=0$ and by (79), $c_{1}=1$. Hence using (67) we get

$$
r^{*}=\frac{2}{3(e-1)}=.387984471 \text {. }
$$

By (78) and (79) we obtain $\gamma=e$ and $c_{1}=e^{-1}$. It follows from Theorem 2.5 in $[12]$ that

$$
r_{U}=\frac{2}{e^{2}}=.270670566 \text {. }
$$

Furthermore the convergence radius by Rheinboldt [8] is given by

$$
r_{R}=\frac{2}{3 \gamma}=.245252961 \text {. }
$$

We conclude that

$$
r_{R}<r_{U}<r^{*}
$$


Hence our results provide a wider choice of initial guesses $x_{0}$ than before. This observation is important in computational mathematics (see also Remark 6). Moreover, since $\ell<\gamma$ our error bounds on the distances $\left\|x_{n}-x^{*}\right\|$ are more precise than the ones using only condition (78) as in [12].

\section{References}

[1] I. K. Argyros, Inexact Newton methods and nondifferentiable operator equations on Banach spaces with a convergence structure, Approx. Theory Appl. 13 (1997), 91-104.

[2] -, On a new Newton-Mysovskii-type theorem with applications to inexact Newtonlike methods and their discretizations, IMA J. Numer. Anal. 18 (1997), 37-56.

[3] I. K. Argyros and F. Szidarovszky, The Theory and Applications of Iteration Methods, CRC Press, Boca Raton, FL, 1993.

[4] P. N. Brown, A local convergence theory for combined inexact-Newton/finite-difference projection methods, SIAM J. Numer. Anal. 24 (1987), 407-434.

[5] N. H. Josephy, Newton's method for generalized equations, Technical Report No. 1965, Math. Res. Center, Univ. of Wisconsin, Madison, WI, 1979.

[6] L. V. Kantorovich and G. P. Akilov, Functional Analysis, Pergamon Press, New York, 1982.

[7] J. S. Pang and D. Chan, Iterative methods for variational and complementarity problems, Math. Program. 24 (1982), 284-313.

[8] W. C. Rheinboldt, An adaptive continuation process for solving systems of nonlinear equations, in: Banach Center Publ. 3, PWN, 1977, 129-142.

[9] S. M. Robinson, Generalized equations, in: A. Bachem et al. (eds.), Mathematical Programming: The State of the Art, Springer, Berlin, 1982, 346-367.

[10] G. Stampacchia, Formes bilinéaires coercitives sur les ensembles convexes, C. R. Acad. Sci. Paris 258 (1964), 4413-4416.

[11] L. U. Uko, Remarks on the generalized Newton method, Math. Program. 59 (1993), 405-412.

[12] - Generalized equations and the generalized Newton method, ibid. 73 (1996), $251-268$.

[13] R. U. Verma, Nonlinear variational and constrained hemivariational inequalities involving relaxed operators, ZAMM 77 (1997), 387-391.

[14] —, A class of projection-contraction methods applied to monotone variational inequalities, Appl. Math. Lett. 13 (2000), 55-62.

[15] —, Generalized multivalued implicit variational inequalities involving the Verma class of mappings, Math. Sci. Res. Hot-Line 5 (2001), 57-64.

Department of Mathematical Sciences

Cameron University

Lawton, OK 73505, U.S.A.

E-mail: ioannisa@cameron.edu

Received on 25.11.2002;

revised version on 12.11.2003 